\title{
CINQUE ETIMOLOGIE VENEZIANE ANTICHE
}

Si raccolgono qui alcune osservazioni etimologiche, che riguardano voci (invero, piuttosto rare) non piu in uso a Venezia, ma bene testimoniate nei documenti antichi. I problemi, che pone questo tipo di ricerca, sono strettamente legati alla limitatezza delle informazioni ricavabili dai testi, alla scarsità di possibili riscontri, che non siano quelli dei vocabolari storici, ristretti alla lingua letteraria, e alla mancanza di tutti $i$ parallelismi, che le parole dialettali solitamente offrono attraverso le raccolte lessicali vernacolari e gli atlanti linguistici.

1. beretin 'bigio, cinerognolo'

La più antica attestazione finora nota di questo aggettivo (presto sostantivato, sottintendendo "panno,), molto diffuso anche in it. (berrettino), ci porta a Venezia. Nell'inventario di una bottega rialtina nel 1225 si trovano "In primis pecias IIII de beretino" (Maggior consiglio 1950: 79), cioe quattro pezze di stoffa di color grigio. E Venezia è indicata come centro di espansione della voce, dopo aver ottenuto, per un passo del Cennini, 1 'ingresso nei vocabolari storici, quando il nome del colore era già entrato da tempo nella pratica commerciale (Pegolotti; ed inoltre Frey 1962: 79 e Rossebastiano Bart 1983: $2,600)$.

La proposta etimologica tradizionale (dal lat. birrus 'rosso, rossiccio') risale al Mussafia 1873:133 (con ampi riferimenti anche alla sua distribuzione geografica) ed è stata via via accettata dal Meyer-Lübke, da C. Merlo, da B. Migliorini e A. Duro, dal Battaglia e da altri ancora. Solo il DEI si è posto contro corrente con la ricostruzione di un lat. "venetinus, dall'agg. venetus 'turchino', ammettendo, tuttavia, una possibile contaminazione con birrus (etimo indicato con perplessitâ da D. Olivieri).

Come già accennato (Cortelazzo-Zolli 1979:133, dove è staccato dal diverso uso in canaglia berettina 'tristo, perfido'), 
si ritiene, invece, che esso non sia altro che l'adattamento di un ar. bāruț̄i, ăgg. di bārụ̆d 'polvere da sparo', come conferma anche Pellegrini 1982: 332-233.

Data l'epoca d'importazione, è escluso il tramite turco col suo prestito barut $\bar{i}$ 'color ardesia, grigio scuro' (Redhouse 1968), al quale è da preferire l'arabo-persiano bāruti 'grigio' (propriamente, 'del colore della polvere da sparo').

2. fax gionda 'far baldoria'

La loc. venez. far gionda 'far baldoria', registrata prima dal Patriarchi nel 1796, assieme al verbo giondàr, di cui gionda è deverbale, poi dal Boerio nel 1829, appartiene ad un compatto filone che da Revine Lago ( $(g)$ ionda: Tomasi 1983) risale verso il bellunese (giondina : Nazari 1884), I'agordino (fa le (s)giónde: Pellegrini 1947-48) e lo zoldano (gionda: Gamba-De Rocco 1972), si attesta bene nel Friuli ( $f a)$ gionde e giondà: Nuovo Pirona 1935) ed arriva fino a Pirano (giondâa: Rosamani 1958).

La sua origine non sembra problematica: un lat. *gaudāre, come variante di gaudēre (Ascoli 1873: 513), è, comunque, preferibile al lungo percorso ipotizzato dal Vidossich 1906: gondolare (simile a gondolare) $\longrightarrow$ *golondare $\longrightarrow$ *glondare $\longrightarrow *$ gion$d \hat{a}$, mentre $i$ passaggi da *gaudạre (cfr. pausāre)—*gauldāre $\longrightarrow$ gioldâa (cfr. polsâ) $\longrightarrow$ gionda (cfr. ponsa) sono abbastanza plausibili, anche se suscitano un interrogativo inquietante: come si spiega il veneziano giondàr? per recente influsso friulano (il suo isolamento e la semantica convengono bene ad una comunita di lavoratori inseriti nella citta lagunare)? o si tratta del fossile di una più larga ed antica palatalizzazione (Schmid), che ha lasciato qualche traccia più cospicua a partire da Revine Lago, come nota G. B. Pellegrini nella prefazione al Tomasi?

In questa serie si inserisce con proprie caratteristiche una variante gianda (egualmente attraverso *gauldāre, ma con diversa soluzione di -aul- : *gialdâa $\longrightarrow$ giandâ), che accomuna Grado e Chioggia. A. C. Marocco (1983: 33) riporta questi esempi d'uso della locuzione gradese fâ gianda: "I xe'ndai a fâ gianda (Sono 
andati a far bisboccia). L'a fato gianda de elo (Ha fatto di lui quello che ha voluto!). Chi vince facilmente nello sport fa gianda dell'avversario o degli avversari. Il leone che aggredisce l'antilope ne fa gianda". Così a Chioggia fare sgianda è 'rompere tutto': qua i a fato sgianda 'qua hanno rotto tutto' (Naccari-Boscolo 1982).

L'alternativa (prestito o palatalizzazione indigena?) si ripropone.

3. polorbo'sciocco'

L'agg. polorbo 'balordo, babbeo, stolido, minchione' era già antiquato agli inizi dell'Ottocento (Boerio 1829).

In realtà, esso si incontra, e con relativa frequenza, nei testi del Cinquecento, specie nel Calmo:

15.51: "questi è fameggi da carezzar, e no certi polorbi che no sa far un sofrito si i no ha tre massere al culo", saltuzza I 3 (c. 5 v);

1552: "là se fa apetito, se fa bon stomego, se paisse, se usa a inscir de polorbi", Rossi 235 ;

1553: "poltron, polorbo, Re de i scagazi", Egloghe II (c. 32); 1556: "de chel polorbo del Rambioso", Travaglia III 12 (c. $51 \mathrm{r}$ ); "Vaga un puoco come se vogia polorbi". Travaglia IV 5 (c. $6.8 \mathrm{v})$.

Credo che si tratti di un Polo (= Paolo, nome di figura nota o inventata) orbo, cioe di uno di quei ciechi che cantavano, accompagnandosi con uno strumento, per le strade della città per qualche quattrino, come è provato diffusamente anche altrove (G. Folena in "Lingua nostra" XVII, 1956, p. 68, nota 10; Pitre 1889, 18, 339, 352) e fino a tempi vicini a noi (Luri di Vassano 1875: 380-381). Che nome e condizione fossero legati è confermato dalla tarda (1604) commedia Tradimento amoroso di B. Maggi, dove interviene un orbo sonatore, chiamato polo orbo ("poco fa sonando con Polorbo", V 5 (c. 53 v)). Il tipo ed il passaggio ad una connotazione negativa rientrano in una casistica diffusamente esaminata da Migliorini 1927: 199-202.

4. sarandégola 'fionda'

Il primo a mettere in discussione l'origine del venez. sa- 
randégola 'fionda' è stato A. Mussafia (1873: 197), il quale, incontratolo in uno dei tanti vocabolari italo-tedeschi del $\mathrm{XV}$ sec., non poteva fare a meno di porlo in connessione con il padov. frandìgolo, con il latino mediev. frandegulum della Historia belli Forojulensis, una cronaca degli avvenimenti accaduti nella seconda metà del Trecento (cit. dal Du Cange), e con l'it. frandibolo (0. Ferrari, 1669).

Possiamo aggiungere, che la voce ricorre, come termine storico, nella Cirurgia universale e pexfetta (Venezia, 1583) di Giovanni Andrea Dalla Croce: "Usavano gli antichi li frandigoli, che a questi tempi sono in uso appresso fanciulli per tirar ... le picciole pietre" (Battaglia 1970). Allo stesso ambiente settentrionale, anzi delle sole venezie, ci riportano altre tre attestazioni in lat. mediev., a Verona nel 1276: "Item statuimus quod nullus, magnus vel parvus prohyceat lapides vel ballotas terre, cum arcu preerio vel cum frandevalo" (Sandri 1940: 432); a Udine nel 1425, che ripete il testo dello statuto di un secolo prima: "Ad corectionem maxime puerorum statutum fuit et reformatum quod nemo ... cum funda sive frandeolo prohicere audeat seu trahere" (Carusi-sella 1930: 81); a Bassano nel 1506: "funda seu frandegolo" (Sella 1944). A questi documenti si può affiancare la conferma del vic. franségolo con la s sonora (Pajello 1896), fxandìgolo ora del vic. rustico (frandigolo, fransègolo: Candiago 1982) e del friul frandéu o sfrandèu, che usò il Bosizio (morto nel 1743), cit. nel Nuovo Pirona 1935, nella sua traduzione dell'Eneide ("Alla' usanza chiargnella pia il frandeu"). La base, chiarissima, è il lat. fundibulus, fundibalus 'balestra' (Diez 1887: 587, REW 3582a, Alessio 1959: 72, Prati 1968) con inserzione di $r$, come nel piem. franda (Nigra 1878: 15).

L'identificazione, apparentemente così scontata, con il tipo parallelo sarandegola, consiste nella presenza di una ssostitutiva di $f-$.

Prima di arrivare ad una proposta, raduniamo la documentazione disponibile sulla variante:

1369: "aliqui pueri ... prohicientes lapides cum sarandegolis" ("Byz. Zeit." XIII, 1904, p.:456); 
1460: "sarandégola: slauder" (Mussafia 1873);

1550: "Perche Follada con un sarandegolo / Cuogoli el trava grossi como ravi" (Caravia, ott. 131);

1553: "No trago mai se no de cerendagolo" (Calmo Egloghe I c. 12);

1553: "Vardeve de sto cerendegolo" (Calmo Rodiana V 65: C. 105);

1867: "Cerendègolo, s.m. Vocabolo antico.... ed è la frombola, detta da noi fionda". (Boerio).

L'alternanza di $c$ e $s$ ci suggerisce di dare la preceden$z a$, nell'evoluzione della voce, alle forme con l'affricata $(c=z)$, ricostruendo questa trafila: f $\mathrm{undibulus}>$ frondigolo > frandigolo > *arandigolo (cfr. l'oscillazione ven. fra fragnocola e faragnócola e fra frangola e faràngola) > *arandìgolo > sarandigolo.

La reciproca sostituzione di $z-e f-$, che era un problema nel 1873, ora è vista chiaramente, come sviluppo abbastanza frequente. Numerosi esempi sono stati raccolti dal Prati (1940: 59) e soprattutto dal Pellegrini (1949:33-34; Pellegrini 1977: 278279).

Questo passaggio presuppone una reazione alla pronuncia $f$ al1'interdentale $\theta$ : cioè, poiche rusticamente si pronunciava, ad esempio, famoro (Oamoro) per zamoro e feliera (Oeliera) per zeliera, indebitamente si risaliva da farandigolo a zarandigolo (sia il Prati 1914: 170, sia il Pellegrini 1977: 278:279 sono, però, dell'opinione che la z sia solo una rappresentazione grafica di $\theta$ ).

Si vuole, con questo, asserire che nel XIV secolo a Venezia sussisteva qualche segno di pronuncia interdentale? Il problema è ancora aperto ( $\mathrm{i}$ veda lo status quaestionis in Pellegrini 1982: 32-33), ma in questo caso si puo pensare, per una parola cosi popolare e 'rustica', anche ad un accatto dalla vicina campagna, tanto più che l'unico manoscritto del vocabolario veneto-tedesco, che accoglie sarandégola, non è immune da numerose tracce di veneto di terraferma (Bart Rossebastiano 1981). Resterebbe la spiegazione della s-per z-, ma oramai è comunemente accettata l'opinione che almeno dal sec. XV l'affricata abbia completamente assunto, per iniziativa dei ceti piu 
bassi della popolazione (e la fionda era diventata gioco di ragazzi e arma di popolani), la pronuncia sibilante, che ha tuttora, e che la conservazione di $z$ (o dei suoi equivalenti $c, \zeta$ ) nella scrittura non sia altro che il frutto di un pigro adequamento ad una antica tradizione grafica (Vidossich 1890-1900: 299-302 e Stussi 1965: LV).

\section{5. vieto 'membro virile'}

Vieto s'incontra due volte in antichi testi veneziani (ma sarebbe, forse, meglio dire, genericamente, veneti) : una prima volta in un vocabolario veneto-tedesco del XV secolo conservato a Heidelberg: "el vieto = der Zers" (Rossebastiano Bart 1983: 2, 303): la seconda volta, ma con tre occorrenze, nelle Dieci tavole di proverbi (1535: D 7, F 8, Q 5): "Do cose inter cetera ha el vecchio che no voria: la carne dura per i denti e il vieto": "Fè conto de lazar el vieto a un sarasin a far questo": "Quando el formento è in la spiga, tira el vieto della figa e non ghe 10 tornar, fin che non n'è campi da vendemar". Un'aitra testimonianza di questa voce si ha in Friuli (Nuovo Pirona 1935), dove vet è oggi forma antiquata, usata dai poeti Nicolò Morlupino (sec. XVI) ed Eusebio Stella (sec. XVII). La base è il lat. $v$ ěctis 'stanga' (REW 9173), in impiego figurato. Altro dei numerosi esempi di metafora sessuale.

\section{Bibliografia}

G. ALESSIO, Nuove etimologie latine e romanze, in: Ioanni Dominico Serra ex munere laeto inferiae. Raccolta di studi linguistici in onore di G. D. Serra, Napóli (Liguori) 1959, pp. 51-104.

G. I. ASCOLI, Saggi ladini, in "Archivio glottologico italiano" I. (1873) 1-556.

A. BART ROSSEBASTIANO, Per la storia dei vocabolari italiano-tedeschi: Localizzazione e datazione di un ramo della tradizione manoscritta, in "La Ricerca dialettale". III (1981) 289-302.

S. BATTAGLIA, Grande dizionario delia lingua italiana, Torino (UTET) I96I e segg.

G. BOERIO, Dizionario del dialetto veneziano, venezia (santini) 1829 .

G. BOERIO, Dizionario del dialetto veneziano ... Venezia (Cecchini) $1867^{3}$. 
A. CALMo, La piacevole et giocosa comedia di M. Andrea Calmo intitolata II Saltuzza, Venezia (Alessi) 1551.

A. CALMO, Le giocose, moderne et facetissime egloghe pastorali, Venezia (Bertacagro) 1553 .

A. CALMO, Rhodiana. Comedia ...., Venezia (Alessi) 1553 .

A. CALMo, Il Travaglia. Comedia di M. Andrea Calmo, Venezia (Alessi) 7556.

E. CANDIAGO, Vocabolario del dialetto vicentino, Vicenza (Cenacolo poeti Dialettali vicentini) 1982.

A. CARAVIA, La verra antiga de Castellani, Canaruoli, e Gnatti, con la morte de Giurco e Gnagni, in lengua brava, i5 50 , s.n.t.

E. CARUST, PISETLLA (ed.), Statuti di Udine del secolo XIV, Milano (Hoepli) 1930 .

M. CORTELAZZO, P. ZOLLT, Dizionario etimologico della lingua italiana, I/A-C, Bologna (zanichelli) 1979.

$D E I=C$. BATTISTI, G. ALESSIO, Dizionario etimologico italiano, Firenze (Barbera) 1950-1957.

DIECI TAVOLE, Opera quale contiene le Diece Tavole de proverbi, sententie, detti, e modi di parlare, che hoggi di da tutthomo nel comun parlare d'Italia si usano, Torino (Cravoto) 1535.

F. DIEZ, Etymologisches wörterbuch der romanischen sprachen, Bonn (Marcus) $1887^{5}$.

H. -J. FREY, Per la posizione lessicale dei dialetti veneti, venezia-Roma (Istituto per la collaborazione culturale) 1962 .

P. LURE DI VASSANO, MOdi di dire proverbiali e motti popolari italiani, spiegati e commentati, Roma lip. Tiberina) 187.5 .

B. MAGGI, I1 tradimento amoroso.Comedia nova di Biagio Maggi, Padova (Bolzetta) 1604 .

Maggior consiglio = Deliberazioni del Maggion consiglio di venezia, I, per cura di $R$. Cessi, Bologna (Zanichelli) 1950 .

A. C. MAROCCO, Forme del dialetto gradese, Poggibonsi (Lalli) 1983.

B. MIGLIORINI, Dal nome proprio al nome comune ..., Genève (O1schki) 1927.

A. MUSSAFIA, Beitrag zur Kunde der norditalienischen Mundarten im XV. Jahrhunderte, Deutsch. Ak. Wien, phil.-hist. $K 1 \ldots, \operatorname{XXII~(1873)~103-228.~}$

R. NACCARI; G. BOSCOLO, VOCabolario del dialetto chioggiotto, Chioggia (Charis) 19.82. 
G. NAZARI, Dizionario beliunese-italiano ...., oderzo (Bianchi) I 884 .

C. NIGRA, Fonetica del dialetto di Val-Soana (Canavese), in "Archivio Glottologico Italiano" III (1878) I-60.

Nuovo Pirona $=$ G. A. PIRONA, E. CARLETTI, G. B. CORGNALI, II nuovo Pirona. Vocabolario friulano, Udine (Bosetti) 1935.

L. PAJELLO, Dizionario vicentino-italiano e italiano-vicentino. I, Vicenza (Brunelio e Pastorio) 1896.

G. PATRIARCHI, Vocabolario veneziano e padovano..., Padova (Coniatil) 1796 .

G. B. PELLEGRINI, Appunti etimologici e lessicali sui dialetti ladino-veneti dell'Agordino, in "Atti dell'Istituto veneto di Scienze, Lettere ed Arti" CVI (1947-48), Parte II, 251-279.

G. B. PELLEGRINI, Lè interdentali nel Veneto, in Atti del Laboratorio ai Fonetica dell'universita di Padova, I (1949) 25-38.

G. B. PELLEGRINI, Studi di dialettologia e filologia veneta, Pisa (Pacini) 19.77.

G. B. PELLEGRINI, Osservazioni di sociolinguistica italiana, in "L'Ttalia dialettale" XLV (1982) I-36.

G. B. PELLEGRINI, Noterelie etimologiche arabo-italiane, in "Rivista "storica calabrese" III (1982) 325-337 (= SCritti linguistici offerti a Gerhard RohIfs nonagenario).

G. PITRE, Usi e costumi, credenze e pregiudizi del popolo siciliano. I, palermo (Clausen) 1889. Nuova ediz.: Roma (Casa Ed. del Libro Italiano) s.d.

A. PRATI, Escursioni toponomastiche nel Veneto, II, in "Bulletin de dialectologie romane"VI (1914) 139-194.

A. PRATI, Voci di gerganti, vagabondi e malviventi studiate nella origine e nella storia, Pisa (Cursi) 1940 .

A. PRATI, Etimologie venete a cura di Gianfranco folena a Giambattista Pellegrini, Venezia-Roma (Istituto per la Collaborazione culturale) 1968 .

REDHOUSE = New Redhouse Turkish-English Dictionary, Istanbul (Redhouse Press) 1968.

$R E W=W . M E Y E R-L \ddot{U} B K E$, Romanisches etymologisches wörterbuch, Heidelberg (winter) $1935^{3}$.

E. ROSAMANT, Vocabolario giuliano, Bologna (Cappelii) 1958 .

A. ROSSEBASTIANO BART, Vocabolari Veneto-Tedeschi del secolo XV, Savigliano (Ed. Savigliano) 1983.

V. ROSSI, Le lettere di messer Andrea Calmo riprodotte sulle stampe migliori con introduzione ed illustrazioni, Torino (Loescher) 1888 . 
G. SANDRI, Gli statuti veronesi del 1276 ..., Venezia (Deputazione di Storia Patria) 1940.

H. SCHMIDT, Über Randgebiete und Sprachgrenzen, in "Vox Romanica" XV 2 (1956) 19-80.

P. SELLA, Glossario latino italiano. Stato della Chiesa-VenetoAbruzzi, Città del Vaticano (Biblioteca Apostolica Vaticana) 1944 .

A. STUSSI, Testi veneziani del Duecento e dei primi del Trecento a cura di Alfredo Stussi, Pisa (Nistri-Lischi), 1965.

G. TOMASI, Dizionario del dialetto bellunese arcaico, Belluno (Istituto Bellunese di Ricerche Sociali e Cultura1i) 1983 .

G. VIDOSSICH, Studi sul dialetto triestino, in "Archeografo triestino" n.s. XXVII (1899-1900) 239-304 e XXVIII (1902) 5-78.

G. VIDOSSICH, Etimologie triestine e istriane, in "Archeografo triestino" XXXI (1906) 71-84.

\footnotetext{
Povzetek

PET STARIH BENEŠKIH ETIMOLOGIJ

$V$ članku so predstavljene etimologije petih beneških izrazov, znanih iz starih tekstov (beretin 'pepelnato siv', 'blago take barve'; far gionda, giondar 'hrupno veseljačiti'; polorbo 'bedak'; sarandegola 'fraća', vieto 'moski ud'), ki pa jih današnji beneški dialekti ne poznajo. Iz tega izvirajo težave pri iskanju etimologij, saj jih ne vsebujejo ne besednjaki posameznih lokalnih govorov, ne lingvistični atlasi.
} 